\title{
A PRÁTICA DA LONGITUDINALIDADE NO ATENDIMENTO À SAÚDE DA CRIANÇA: COMPARAÇÃO ENTRE MODELOS ASSISTENCIAIS DISTINTOS*
}

Vanessa Comassetto Oliveira', Maria de La Ó Ramallo Veríssimo²

${ }^{1}$ Enfermeira. Doutoranda em Enfermagem. Escola de Enfermagem da Universidade de São Paulo. Curitiba-PR-Brasil. ${ }^{2}$ Enfermeira. Doutora em Enfermagem. Escola de Enfermagem da Universidade de São Paulo. São Paulo-SP- Brasil.

RESUMO: Avaliou-se a orientação dos serviços primários de saúde sob o foco da longitudinalidade, na atenção à criança. Estudo transversal e abordagem quantitativa em unidades com Estratégia Saúde da Família e o modelo tradicional nas Unidades Básicas de Saúde. Os dados levantados por entrevistas, entre junho e julho de 2012, com famílias de crianças ( $\mathrm{n}=482$ ) foram analisados conforme o Primary Care Assesment Tool. Constatou-se que a relação pessoal de longa duração entre os profissionais de saúde e os pacientes e familiares é fragilizada em ambos os modelos de assistência. As Estratégia Saúde da Família obtiveram escore acima do valor de corte em dois de 11 itens do atributo longitudinalidade. As Unidades Básicas de Saúde tiveram todos os itens com escores abaixo do valor. O princípio da longitudinalidade não se mostrou incorporado, a sua prática como princípio do Sistema Único de Saúde ainda permanece como desafio em ambos os modelos assistenciais.

DESCRITORES: Atenção primária à saúde; Saúde da criança; Avaliação em saúde.

\section{THE PRACTICE OF LONGITUDINALITY IN ATTENDANCE OF CHILDREN'S HEALTH: COMPARISON BETWEEN DISTINCT CARE MODELS}

\begin{abstract}
The orientation of the primary healthcare services was evaluated in the perspective of longitudinality in children's healthcare. A transversal study with a quantitative approach was undertaken in the units with the Family Health Strategy and of the traditional model in the Primary Care Units. The data were raised through interviews held between June and July 2012, with families of children $(\mathrm{n}=482)$, and were analyzed using the Primary Care Assessment Tool. It was observed that long-duration personal relationships between the health professionals and the patients and family members is weakened in both models of care. The units of the Family Health Strategy obtained a score above the cut-off value in two of 11 items of the attribute 'longitudinality'. The Primary Healthcare Units had all the items with scores below the value. The principle of longitudinality was not shown to be incorporated, and its practice, as a principle of the Unified Health System, continues to be a challenge in both the models of care.
\end{abstract}

DESCRIPTORS: Primary Health Care; Children's health; Health evaluation.

\section{LAPRÁCTICADELALONGITUDENELATENDIMIENTO A LA SALUD DEL NIÑO: COMPARACIÓN ENTRE MODELOS ASISTENCIALES DISTINTOS}

RESUMEN: Se evaluó la orientación de los servicios primarios de salud bajo el ángulo de longitud en la atención al niño. Es un estudio transversal de abordaje cuantitativo en unidades con Estrategia Salud de la Familia y en modelo tradicional en las Unidades Básicas de Salud. Los datos obtenidos por entrevistas, entre junio y julio de 2012, con familias de niños $(\mathrm{n}=482)$, fueron analizados de acuerdo a Primary Care Assesment Tool. Se constató que la relación personal de larga duración entre los profesionales de salud y los pacientes y familiares es fragilizada en ambos los modelos de asistencia. Las Estrategias Salud de la Familia obtuvieron score mayor que el valor de corte en dos de 11 ítenes del atributo longitud. Las Unidades Básicas de Salud tuvieron todos los ítenes con scores abajo del valor. El principio de la longitud no se ha mostrado agregado; su práctica como principio del Sistema Único de Salud todavía permanece como desafío en ambos los modelos asistenciales.

DESCRIPTORES: Atención primaria a la salud; Salud del niño; Evaluación en salud.

\footnotetext{
* Artigo extraído da dissertação de mestrado intitulada:Avaliação da Atenção Primária à Saúde da Criança no município de Colombo - PR. Escola de Enfermagem da Universidade de São Paulo, 2012.
}

\section{Autor Correspondente:}

Vanessa B. Comassetto A. Oliveira

Escola de Enfermagem da Universidade de São Paulo

Rua Major Vicente de Castro, 2138 - 81030-020 - Curitiba-PR-Brasil

E-mail: vancomassetto@hotmail.com
Recebido: 04/02/2014

Finalizado: 14/10/2014 


\section{INTRODUÇÃO}

O tema central deste estudo é o princípio da longitudinalidade, com enfoque na saúde da criança. Historicamente, a criança foi prioridade nas políticas públicas de saúde. Os perfis de morbimortalidade infantil eram resistentes às ações realizadas. É claro que as condições de saúde estavam intimamente relacionadas às condições de vida dessa população, portanto, ações como aumento da cobertura vacinal, melhoria das condições de saneamento básico e de nutrição e a introdução e expansão da terapia de reidratação oral foram marcos decisivos na mudança do perfil de morbimortalidade nas últimas décadas ${ }^{(1)}$.

Torna-se necessário refletir sobre os atuais indicadores para auxiliar a compreensão da saúde da criança na sociedade contemporânea. A cada ano, cerca de quatro milhões de recémnascidos morrem antes de completar 28 dias de vida e, nos países em desenvolvimento, o risco de uma criança morrer é 14 vezes maior do que em um país desenvolvido. Entre 1990 e 2012 a taxa de mortalidade infantil caiu de 13 milhões para 6,9 milhões, atingindo em 33\% a meta do milênio de redução de mortalidade ${ }^{(2)}$. Ao mesmo tempo em que a mortalidade persiste como um desafio, ressalta-se a

importância do cuidado integral à criança na primeira infância, que tem influência no sucesso escolar, no desenvolvimento da resiliência e autoestima, na formação de relações e autoproteção, visando a sua independência ${ }^{(1: 24)}$.

A conjuntura social do sistema de saúde envolve a implantação real do Sistema Único de Saúde (SUS), criado pela Constituição Federal do Brasil de 1988 e tem como diretrizes a universalização do direito à saúde, a integralidade das ações preventivas e curativas, a descentralização dos serviços e a participação social. Ainda, a fim de se adequar às determinações contidas na Declaração da Alma Ata, o Brasil adotou a Atenção Primária à Saúde (APS) como estratégia para organizar o sistema, sendo esta, portanto, considerada como parte integral do sistema de saúde que enfoca o indivíduo, a família e a comunidade. A APS busca aproximar a atenção à saúde do local onde as pessoas vivem e trabalham, construindo o primeiro elemento de um processo de educação continuada à saúde ${ }^{(3)}$.

O SUS do Brasil, como aparato jurídico-legal é um dos mais avançados no mundo, porém quando se contextualizam as dimensões sócioculturais, políticas e econômicas na qual esse sistema se concretiza, surgem contradições de diversas ordens. Dentre as mais importantes destaca-se a ideologia do estado como provedor e de direitos para uma massa de cada vez mais excluídos ${ }^{(4)}$. Portanto, avançar nos mecanismos do SUS é entendido como uma forma processual na busca de avanços na consolidação de um sistema de saúde inserido na realidade social brasileira ${ }^{(2)}$.

Ainda em se tratando do SUS, uma das medidas do poder público foi a criação da Estratégia Saúde da Família (ESF), entendida como capaz de compreender e operacionalizar a abordagem integral do processo saúdedoença e responder de forma mais efetiva aos problemas de saúde da população. É um processo dinâmico que permite a implementação dos princípios e diretrizes da Atenção Primária, devendo se constituir como ponto fundamental para a organização da rede de atenção. Entretanto, reconhecemse dificuldades para adequar esse modelo assistencial aos princípios como o aumento de vínculo entre os serviços de saúde e a população, ou seja, a longitudinalidade ${ }^{(5)}$.

Longitudinalidade é reconhecido como um dos atributos essenciais da APS ${ }^{(6)}$. Também conhecida como continuidade, é definida como o vínculo com o serviço ao longo do tempo, de forma que, quando uma nova demanda surge, esta seja atendida de forma mais eficiente. O alcance da Atenção Primária implica a existência de um local, um indivíduo ou uma equipe de indivíduos associados que sirvam como fonte de atenção por um determinado período de tempo, independente da presença ou ausência de problemas relacionados à saúde ${ }^{(7)}$. Ter a atenção longitudinal significa que aqueles indivíduos na população identificam uma fonte de atenção como "sua", que os prestadores de serviço reconhecem a existência de um contrato formal ou informal para ser a fonte habitual da atenção orientada para a pessoa (e não para a doença) $)^{(6-7)}$. 
Estudos revelam inúmeros benefícios da presença da longitudinalidade na atenção prestada à criança nos serviços de saúde, como a compreensão dos históricos de doença do paciente, a facilidade em prescrever ações de prevenção, precisão nos diagnósticos e redução de custos ao sistema público de saúde ${ }^{(7)}$.

Esta pesquisa foi realizada no município de Colombo, estado do Paraná, o qual conta com dois modelos de assistência na Atenção Primária, as Unidades Básicas de Saúde, as quais seguem ao modelo tradicional da atenção programática, e as Unidades com $\mathrm{ESF}^{(8)}$. Uma vez que ambas são veículos do SUS, a longitudinalidade é um dos princípios que deveria nortear a atenção nelas prestada.

Portanto, o propósito deste estudo foi avaliar e comparar a presença e a extensão do atributo longitudinalidade nos serviços das UBS e das ESF do município de Colombo, estado do Paraná.

\section{MÉTODO}

Trata-se de estudo com delineamento transversal, de abordagem quantitativa, realizado no município de Colombo, estado do Paraná. Este concentra uma população de 247.268 habitantes, sendo que a estimativa é que $8,58 \%$ correspondam a crianças de zero a cinco anos. Para atender a essa população, a Secretaria Municipal de Saúde disponibiliza 21 unidades de saúde, sendo que nove são integradas à ESF e as demais são Unidades Básicas tradicionais ${ }^{(8)}$.

A população do estudo constituiu-se de famílias de crianças de 0 a 1 ano completo, cadastradas e assíduas às atividades das unidades de saúde do município, pois nesta faixa etária foram realizadas as consultas mais recentemente, o que favorece que o principal cuidador possa responder as informações mais atualizadas. Os critérios de exclusão foram crianças atendidas na rede particular de saúde, com endereço inexistente ou não localizado, crianças adotadas ou falecidas e número de atendimentos na unidade de saúde inferior a três durante o primeiro ano de vida.

Com base no total da população da pesquisa, realizou-se o cálculo de amostra com
95\% de confiança e 3\% de erro máximo de estimativa. Foram entrevistadas 235 famílias, representadas pelo cuidador principal da criança, pertencentes às unidades Estratégia Saúde da Família e 247 famílias das Unidades Básicas de Saúde, totalizando 482 sujeitos.

Para a coleta de dados, 12 acadêmicos de enfermagem cadastrados em programas de iniciação científica de uma universidade pública, com projetos naquela localidade, foram treinados para realizarem as entrevistas, as quais ocorreram em junho e julho de 2012. As entrevistas foram individuais com o apoio do instrumento Primary Care Assessment Tool (PCATool) versão criança, já validado no Brasil( ${ }^{(9)}$.

A análise dos dados se deu conforme recomendação do PCATool, cuja aplicação pontua escores para os itens do instrumento que variam de 0 a 10, sendo considerado ideal para Atenção Primária à Saúde valores acima da média $6,6^{(9)}$.

O estudo teve aprovação pelo Comitê de Ética em Pesquisas da Escola de Enfermagem da Universidade de São Paulo e das instâncias competentes da Secretaria Municipal de Saúde de Colombo e todas as famílias responderam somente após concordância e assinatura do termo de consentimento livre e esclarecido. A pesquisa foi apreciada com o registro $\mathrm{CAAE}$ número 02559112.2.0000.5392 e se cumpriu os rigores e princípios éticos estabelecidos pela Resolução 196/96.

\section{RESULTADOS}

Na Tabela 1, encontra-se o perfil dos responsáveis pelas crianças bem como dados sobre tipo e número de atendimentos recebidos nos serviços de saúde analisados.

Da totalidade dos participantes da pesquisa, 48,8\% utilizam os serviços da rede de Atenção Básica em Saúde na modalidade ESF e 51,2\% utilizam serviços das UBS.

A Tabela 2 expressa os escores dos itens que compõem o atributo Longitudinalidade, aferidos a partir da percepção dos cuidadores das crianças atendidas nas unidades com Estratégia Saúde da Família e nas Unidades Básicas de Saúde, as quais alcançaram escores médios de 5,4 e 3,4 respectivamente. 
Tabela 1 - Características dos responsáveis pelas crianças atendidas, número de consultas durante o primeiro ano de vida e tipo de atendimento recebido no serviço, segundo o tipo de Unidade de Saúde. Colombo, Paraná, 2012

\begin{tabular}{|c|c|c|c|c|c|}
\hline \multirow{2}{*}{ Variáveis } & \multicolumn{2}{|c|}{$\operatorname{ESF}(n=235)$} & \multicolumn{2}{|c|}{ UBS $(n=247)$} & \multirow{2}{*}{$\begin{array}{c}\text { Valor } \\
\text { "p" }\end{array}$} \\
\hline & $\mathbf{n}$ & $\%$ & $\mathbf{n}$ & $\%$ & \\
\hline \multicolumn{6}{|c|}{ Parentesco com a criança } \\
\hline Pai & 19 & 8,1 & 14 & 5,7 & 0,2936 \\
\hline Mãe & 187 & 79,6 & 209 & 84,6 & 0,1484 \\
\hline Avó & 28 & 11,9 & 23 & 9,3 & 0,3540 \\
\hline Tia & 01 & 0,4 & 01 & 0,4 & 0,9592 \\
\hline \multicolumn{6}{|l|}{ Sexo do cuidador } \\
\hline Masculino & 19 & 8,1 & 14 & 5,7 & 0,2936 \\
\hline Feminino & 216 & 91,9 & 233 & 94,3 & 0,2936 \\
\hline \multicolumn{6}{|c|}{ Escolaridade do cuidador } \\
\hline Não alfabetizado & 08 & 3,4 & 12 & 4,9 & 0,4221 \\
\hline Alfabetizado & 227 & 96,6 & 235 & 95,1 & 0,3883 \\
\hline \multicolumn{6}{|l|}{ Idade do cuidador } \\
\hline 15 à 20 anos & 72 & 30,6 & 166 & 67,2 & 0,00001 \\
\hline 20 à 30 anos & 146 & 62,1 & 12 & 4,9 & 0,00001 \\
\hline 30 à 40 anos & 11 & 4,7 & 65 & 26,3 & 0,00001 \\
\hline 40 à 50 anos & 03 & 1,3 & 02 & 0,8 & 0,2268 \\
\hline 50 à 60 anos & 02 & 0,9 & 00 & 0,0 & 0,5969 \\
\hline$>60$ anos & 01 & 0,4 & 02 & 0,8 & 0,5969 \\
\hline \multicolumn{6}{|c|}{ Atividades frequentadas pela criança } \\
\hline Consulta & 29 & 12,3 & 37 & 15,0 & 0,3998 \\
\hline $\begin{array}{l}\text { Consulta e } \\
\text { vacina }\end{array}$ & 206 & 87,7 & 210 & 85,0 & 0,3364 \\
\hline \multicolumn{6}{|c|}{ Número de consultas da criança } \\
\hline Três & 189 & 80,4 & 239 & 96,8 & 0,00001 \\
\hline Quatro & 41 & 17,5 & 08 & 3,2 & 0,00001 \\
\hline Cinco & 05 & 2,1 & 00 & 0,0 & 0,0216 \\
\hline
\end{tabular}


Tabela 2 - Valores médios, respectivos erros-padrão (EP) e valor da significância estatística do teste t para cada item que compõe o atributo Longitudinalidade, segundo o tipo de serviço de saúde. Colombo, 2012

\begin{tabular}{|c|c|c|c|c|c|}
\hline \multirow{3}{*}{ Indicadores } & \multirow{2}{*}{\multicolumn{2}{|c|}{$\begin{array}{c}\text { ESF } \\
(n=235)\end{array}$}} & \multirow{2}{*}{\multicolumn{2}{|c|}{$\begin{array}{c}\text { UBS } \\
(n=247)\end{array}$}} & \multirow{3}{*}{ Teste $t$} \\
\hline & & & & & \\
\hline & Média & Ep* & Média & Ep* & \\
\hline $\begin{array}{l}\text { É o mesmo profissional } \\
\text { que atende a criança } \\
\text { no serviço }\end{array}$ & 7,0 & 0,166 & 2,8 & 0,145 & $<0,01$ \\
\hline $\begin{array}{l}\text { Pode telefonar para } \\
\text { falar com o profissional } \\
\text { no serviço }\end{array}$ & 3,7 & 0,148 & 2,6 & 0,144 & $<0,0001$ \\
\hline $\begin{array}{l}\text { O profissional entende } \\
\text { o que você diz ou } \\
\text { pergunta }\end{array}$ & 7,3 & 0,153 & 4,3 & 0,159 & $<0,01$ \\
\hline $\begin{array}{l}\text { O profissional responde } \\
\text { suas perguntas de } \\
\text { maneira que você } \\
\text { entenda }\end{array}$ & 4,6 & 0,159 & 4,1 & 0,161 & 0,018601 \\
\hline $\begin{array}{l}\text { O profissional lhe dá } \\
\text { tempo suficiente para } \\
\text { falar sobre os problemas } \\
\text { ou preocupações }\end{array}$ & 3,6 & 0,165 & 2,3 & 0,142 & $<0,0001$ \\
\hline $\begin{array}{l}\text { Você se sente a } \\
\text { vontade para falar } \\
\text { dos problemas de sua } \\
\text { criança ao profissional }\end{array}$ & 5,7 & 0,188 & 5,4 & 0,189 & 0,195223 \\
\hline $\begin{array}{l}\text { O profissional conhece } \\
\text { a criança como pessoa } \\
\text { mais do que como um } \\
\text { problema de saúde }\end{array}$ & 4,5 & 0,190 & 3,1 & 0,151 & $<0,0001$ \\
\hline $\begin{array}{l}\text { O profissional conhece } \\
\text { a história clínica da } \\
\text { criança }\end{array}$ & 5,2 & 0,193 & 2,7 & 0,143 & $<0,0001$ \\
\hline $\begin{array}{l}\text { O profissional sabe dos } \\
\text { medicamentos que a } \\
\text { criança toma }\end{array}$ & 5,9 & 0,178 & 4,4 & 0,178 & $<0,0001$ \\
\hline $\begin{array}{l}\text { O profissional se } \\
\text { reuniria com sua família } \\
\text { se fosse necessário }\end{array}$ & 6,0 & 0,175 & 2,9 & 0,145 & $<0,01$ \\
\hline $\begin{array}{l}\text { Você mudaria para } \\
\text { outro serviço de saúde } \\
\text { se fosse fácil }\end{array}$ & 5,7 & 0,192 & 2,4 & 0,138 & $<0,01$ \\
\hline Escore Geral & 5,4 & 0,189 & 3,4 & 0,166 & $<0,0001$ \\
\hline
\end{tabular}

*Ep = Erro Padrão 


\section{DISCUSSÃO}

Nesse estudo, identificou-se que o perfil dos principais responsáveis das crianças que compuseram a amostra é semelhante nas Unidades Básicas de Saúde e nas Unidades Estratégia Saúde da Família, sendo as mães as principais responsáveis, seguidas das avós, corroborando outros estudos ${ }^{(10)}$.

Também há semelhança entre as Unidades no que se refere escolaridade do responsável, com a grande maioria, considerada alfabetizada. Este perfil está de acordo com o perfil social e demográfico do município, o qual indica que o índice de analfabetismo tem diminuído desde $2006^{(8)}$.

A principal diferença entre os perfis dos entrevistados nas unidades pesquisadas é referente à idade dos responsáveis pelas crianças. As unidades ESF apresentam um perfil com maioria de responsáveis entre 20 e 30 anos, enquanto nas UBS a idade prevalente está abaixo dos 20 anos, sendo a idade mínima de 15 anos. Admitese pensar que essa diferença em consonância com o perfil prevalente de mães, pode indicar um fator de alto índice de maternidade precoce nas UBS. Estudo publicado em 2011 ${ }^{(11)}$ indicou a gravidez na adolescência como um problema emergente no município de Colombo, entretanto este estudo não diferencia os índices segundo o tipo de unidade de saúde de referência.

No que se refere às atividades frequentadas pela criança, há semelhança em ambas as unidades, destacando-se as consultas e vacinas. O Plano Municipal de Saúde do município ${ }^{(8)}$ lista os procedimentos executados em ambas as unidades para as crianças segundo parâmetros de produtividade e informações do Departamento de Atenção Básica da Secretaria Municipal de Saúde, o qual informa ações tais como: administração de medicamentos, curativos, nebulização, terapia de reidratação oral, atividades educativas, coleta de sangue, puericultura, consultas de enfermagem, vacinação, entre outras.

Observa-se que a população reconhece e utiliza as consultas e os serviços de vacinação disponibilizados pela Unidade de Saúde. Embora as demais ações não tenham sido citadas diretamente, é possível que sejam consideradas, pelos usuários, como inerentes às atividades da consulta.
Sobre o número de consultas frequentadas pelas crianças durante o primeiro ano de vida, observou-se que nas Unidades ESF o máximo foi cinco e, nas UBS, quatro. Entretanto, em ambas o predomínio ficou em três consultas. Considerando-se que, no município, recomendase que sejam feitas oito consultas durante o primeiro ano de vida, pois, como preconizado pelo Ministério da Saúde ${ }^{(12)}$ o calendário de consultas para o controle do crescimento e desenvolvimento, de crianças sadias, pressupõe a atuação de toda a equipe de atenção à criança, de forma intercalada, possibilitando ampliação na oferta de atendimentos em todas as unidades de saúde. Nesse sentido, considera-se necessária uma estratégia para aumentar a frequência às consultas em ambas as unidades.

Quanto à continuidade do atendimento pelo mesmo profissional, apenas as ESF estão de acordo com as definições de APS, o qual é definido como o acompanhamento por um mesmo profissional ou não, de um problema específico do paciente e a sucessão de eventos entre uma consulta e outra, bem como os mecanismos de transferência de informações entre um profissional e outro. Estima-se que haja uma rotatividade maior de funcionários nas unidades tradicionais, o que pode justificar a baixa média nas UBS. De acordo com o Conselho Nacional de Secretários de Saúde (CONASS), a rotatividade de profissionais nas equipes de saúde é considerado um dos grandes desafios para a prática do atendimento longitudinal nos serviços de saúde do Brasil ${ }^{(13)}$.

Ambos os modelos de atendimento tiveram baixos escores quanto à possibilidade de contato telefônico com o profissional de saúde, ou seja, o contato não pessoal não é uma prática comum. Esse resultado propicia discutir a necessidade do usuário se dirigir até a unidade de saúde para poder sanar quaisquer dúvidas referentes à saúde da criança, o que pode ser um dos fatores para o acúmulo de filas no aguardo de atendimento nas unidades.

Diferentemente das UBS, as unidades ESF obtiveram escores adequados quando avaliadas se o profissional entende o que o responsável pela criança diz ou pergunta. Esta característica facilita a comunicação entre profissionais de saúde e usuários e gera satisfação no atendimento prestado. Mas, sabe-se que a comunicação é uma 
via dupla, e os dois modelos de atendimento não atingiram o escore mínimo quanto ao tempo dedicado pelos profissionais para entender as dúvidas dos familiares nem quanto a sua expressão de forma compreensível ao responder os questionamentos feitos. Verificou-se, portanto, que a comunicação ainda é uma característica falha em ambas as unidades de saúde, conforme observado em estudos similares ${ }^{(14)}$.

Outro aspecto proporcionado pela longitudinalidade é a sensação de liberdade do usuário para falar dos problemas da criança ao profissional de saúde, fundamental para o estabelecimento de vínculo, que não alcançou valor satisfatório em ambos os tipos de serviço. Assim, mesmo a continuidade do atendimento mais efetiva nas ESF não produz nos usuários tal sensação, o que pode prejudicar a vinculação ao serviço, sendo que a ausência de vínculo desfavorece uma relação de confiança entre as partes do processo $^{(6)}$.

A similaridade nos baixos escores dos itens relativos ao domínio do profissional sobre a situação de saúde-doença da criança sugere prevalência de um atendimento focado na doença em ambos os tipos de serviço. Sugere-se que este achado esteja em consonância com a formação dos profissionais cujos cursos não reiteram a devida importância da medicina preventiva e de conceitos de promoção da saúde ${ }^{(13)}$. Estudos destacam que um dos nós mais críticos citado pelos gestores da APS é a falta de profissionais especializados e com capacidade técnica em saúde da família ${ }^{(14-15)}$.

Este mesmo nó citado pelos gestores em estudos da área pode indicar uma das justificativas para as unidades ESF e as UBS não estarem de acordo com os preceitos da APS quando os responsáveis das crianças são questionados se o profissional de saúde se reuniria com a família caso fosse necessário. Pode-se compreender também que o acumulo da demanda de serviços favorece que não haja disponibilidade dos profissionais para ações diferentes das usualmente realizadas ${ }^{(14)}$.

Por fim, a avaliação do atributo longitudinalidade questiona se o usuário trocaria de serviço de saúde se esse processo fosse fácil. Observa-se que os escores encontrados nos dois modelos de unidade são desfavoráveis, uma vez que os responsáveis das crianças optariam pela troca de serviço.
Analisando, de maneira geral, nota-se a partir dos resultados desta pesquisa que as unidades com ESF e as UBS não estão de acordo com os critérios da APS quando analisadas sob o foco da longitudinalidade.

\section{CONCLUSÃO}

A constante avaliação dos serviços de saúde na rede básica é um dos fundamentos que compõe a APS. Neste sentido, considera-se que os resultados encontrados em estudos de avaliação da APS em todas as esferas de atendimento da rede básica contribuem significativamente para a busca da superação das dificuldades. Transformar a saúde da família de um programa a uma estratégia, dentro da política nacional, exige a permanência e a atualização desses estudos.

Constatou-se que o atributo longitudinalidade precisa ser reforçado em todos os modelos de unidades de saúde, pois ambos mostraramse distantes dos pressupostos da APS. Esta necessidade se dá a fim de otimizar aspectos que ainda desfavorecem a qualidade da assistência prestada na rede básica. Quando um serviço não é fundamentado pela longitudinalidade, observamse aspectos como: ocorrências de tratamentos incompletos, utilização inadequada e incompleta dos serviços de saúde focados nas ações de prevenção, aumento das hospitalizações, redução da capacidade dos profissionais em avaliar adequadamente as necessidades das pessoas e das comunidades, integralidade e coordenação das ações de serviço desfavoráveis ou inexistentes e redução na satisfação dos usuários.

A rotatividade dos profissionais das equipes de saúde e a falta de capacitação aos que estão inseridos no sistema constituem-se como principais desafios da longitudinalidade na APS.

\section{REFERÊNCIAS}

1. Saparolli ECL, Adami NP. Avaliação da estrutura destinada à consulta de enfermagem à criança na atenção básica. Rev. Esc. Enferm. USP. [Internet] 2010;44(1) [acesso em 21 out 2012]. Disponível: http:// www.revistas.usp.br/reeusp/article/view/40512

2. Ministério da Saúde (BR). Secretaria de Atenção à Saúde. Departamento de Ações Programáticas e Estratégicas. Agenda de Compromisso para a Saúde 
Integral da Criança e redução da mortalidade infantil. Brasilia; 2012.

3. Organização Pan-Americana da Saúde (OPAS); Organização Mundial da Saúde (OMS). Renovação da Atenção Primária em Saúde nas Américas: documento de posicionamento da OPAS/OMS [Internet], 2007 [acesso em 13 abr 2012]. Disponível: http://www. uepa.br/portal/downloads/PETSAUDE2012/REF_ BIBLIOGRAFICAS/renovacao_atencao_primaria_ saude_americas.pdf

4. Sllva LOL, Dias CA, Soares MM, Rodrigues SM. Acessibilidade ao serviço de saúde: percepções dos usuários e profissionais de saúde. Cogitare enferm. [Internet] 2011;16(4) [acesso em 3 mar 2013]. Disponível: http://ojs.c3sl.ufpr.br/ojs2/index.php/ cogitare/article/view/25434/17053

5. Macinko J, Almeida C, Oliveira ES, Sá PK. Organization and delivery of primary health care services in Petropolis, Brazil. Int. j. health plann. manag. [Internet] 2004;19(4) [acesso em 23 nov 2012]. Disponível: http:// www.ncbi.nlm.nih.gov/pubmed/15688875

6. Starfield B, Shi L, Macinko J. Contribution of primary care to health systems and health. Milbanq Q. [Internet] 2005;83(3) [acesso em 15 jan 2013]. Disponível: http:// www.ncbi.nlm.nih.gov/pubmed/16202000

7. Shi L, Starfield B. Primary care, income inequality and self-rated health in United States: a mixed level analysis. Int J Health Serv. [Internet] 2000;30(3) [acesso em 2 dez 2012]. Disponível: http://www.ncbi.nlm.nih. gov/pubmed/11109180

8. Colombo. Secretaria Municipal de Saúde. Plano Municipal de Saúde 2010-2013. Colombo (PR), 2010.

9. Ministério da Saúde (BR). Departamento de Atenção Básica. Secretaria de Atenção à Saúde [Internet]. Manual do Instrumento de Avaliação da Atenção Primária à Saúde: Primary Care Assessment Tool PCATool-Brasil. Brasília: Ministério da Saúde; 2010 [acesso em 2 dez 2012]. Disponível: http://bvsms. saude.gov.br/bvs/publicacoes/manual_avaliacao_ pcatool_brasil.pdf

10. Santos MA, Henrique VC, Silva VC. A compreensão das mães acerca da consulta de puericultura numa unidade de saúde da família. Revista Rede de Cuidado em Saúde. [Internet] 2009;3(3) [acesso em 4 abr 2012]. Disponível: http://publicacoes.unigranrio.edu.br/index. php/rcs/article/viewFile/680/565

11. Wolff LDG, Castanharo RCT. A percepção de adolescentes gestantes acerca dos papéis ocupacionais e do futuro materno [dissertação]. Curitiba (PR): Universidade Federal do Paraná; 2011.

12. Ministério da Saúde (BR). Centro de Documentação. Assistência integral a saúde da criança: ações básicas
[Internet]. Série B: Textos Básicos de Saúde, 7. Brasília; 1984 [acesso em 4 abr 2012]. Disponível: http://bvsms. saude.gov.br/bvs/publicacoes/assistencia_integral_ saude_crianca.pdf

13. Brasil. Conselho Nacional de Secretários de Saúde - CONASS. Coleção Pró Gestores - Para entender a gestão do SUS. Atenção Primária e Promoção da Saúde. Brasília; 2007

14. Giovanella L, Lobato LCL, Carvalho Al, Conill EM, Cunha EM. Sistemas municipais de saúde e a diretriz da integralidade da atenção: critérios para avaliação. Saude em debate. [Internet] 2002;26(3) [acesso em 30 set 2012]. Disponível: http://bases.bireme.br/cgibin/ wxislind.exe/iah/online/?IsisScript=iah/iah

15. Cunha EM. Vínculo Longitudinal na Atenção Primária: avaliando os modelos assistenciais do SUS [tese]. Rio de Janeiro (RJ): Fundação Oswaldo Cruz; 2009. 